\section{Irritable bowel syndrome subtype screening characteristics: constipation subtype patient profiles explored}

\author{
Joyce K. Anastasi, Bernadette Capili, \\ Jessica Quinn, Donald J. McMahon, \\ Colin Scully \\ Division of Special Studies in Symptom \\ Management, New York University, \\ New York, NY, USA
}

\section{Abstract}

Irritable bowel syndrome (IBS), a chronic disorder, greatly impacts the lives of those affected. Approximately one quarter of the North American population suffers from this condition that includes abdominal pain, discomfort and alterations in normal bowel function. Constipation-subtype IBS (IBS-C) is often found to be a prevalent subtype; however, a lack of knowledge and effective treatment options remains surrounding this disorder. In the course of an ongoing study conducted by our research team on the use of acupuncture and moxibustion in the management of IBS symptoms, a considerable number of screened patients have met the widely accepted Rome III criteria for IBS-C.

\section{Introduction}

Irritable bowel syndrome (IBS) is a chronic functional bowel disorder that involves abdominal pain or discomfort with associated changes in bowel movements. ${ }^{1}$ Abdominal pain is a common complaint in $\mathrm{IBS},{ }^{2}$ leading patients to seek a diagnosis and treatment. Providers often base a diagnosis of IBS on a thorough patient history, symptom-based criteria and consideration of specific alarm symptoms. ${ }^{3}$ Alarm symptoms, such as rectal bleeding, unintended weight loss or fever may be indicative of a serious underlying condition. Several multinational committees have worked in collaboration to develop the ROME criteria, which provides a standard framework for the inclusion of patients in diagnostic and therapeutic trials of IBS, and more recently for patients in clinical practice. ${ }^{3}$ According to the ROME III criteria, a diagnosis of IBS can be made if a patient experiences recurrent abdominal pain or discomfort at least three days a month during the past three months with symptom onset at least six months prior to diagnosis. ${ }^{1}$ The abdominal pain or discomfort must be associated with at least two of the following: i) improvement with defecation, ii) onset associated with a change in frequency of stool, and/or iii) onset associated with a change in form or appearance of stool. ${ }^{1}$

It is estimated that $3 \%$ to $20 \%$ of the North American population is affected by IBS regardless of race or geographical location, although many studies have found a higher prevalence in women. ${ }^{1,4}$ IBS has an adverse impact on quality of life; affecting work absenteeism, social interactions, sleep patterns and everyday activities. ${ }^{5-7}$ Additionally, the direct and indirect costs of IBS are estimated to exceed $\$ 20$ billion annually. ${ }^{5}$

There are four subtypes of IBS: constipation subtype IBS (IBS-C), diarrhea subtype IBS (IBS-D), mixed IBS (IBS-M), and unsubtyped IBS (IBS-U). ${ }^{1}$ IBS-C is characterized by at least $25 \%$ of bowel movements being hard or lumpy and less than $25 \%$ of bowel movements being loose or watery. ${ }^{1}$ The prevalence of IBS-C has been found to be among the highest of the four subtypes in multiple population-based studies. ${ }^{8}$ Recent studies in Korea, Malaysia and Greece found IBS-C in over half of the patients, $75 \%$ of patients, and as the most common subtype, respectively. 4

Despite the prevalence and bothersome nature of IBS, specifically IBS-C, a dearth of knowledge remains surrounding the disorder and effective treatment options are lacking. ${ }^{9,10}$ The selection of over-the-counter medications claiming to treat the symptoms of IBS is vast and patients often utilize these drugs in an attempt to self-medicate, but with undocumented results. Other treatment options include: lifestyle and dietary changes, psychotherapy, prescription drugs, and complementary and alternative medicine (CAM). ${ }^{1,4}$ Given the limited number of treatments available for IBS, many patients turn to CAM. ${ }^{11} \mathrm{CAM}$ consists of a variety of treatments, examples of which include yoga, herbs, acupuncture, etc., and are often used in addition to conventional medicine. ${ }^{11}$ It is most commonly utilized by patients with a chronic condition for which current conventional medical treatments are not satisfactory. 11 The use of CAM in patients in the United States with functional bowel disorders, including IBS, is reported to be between $35 \%$ to $50 \%$. 6,12

CAM offers a variety of treatment approaches, including traditional Chinese medicine (TCM), a system of medicine that has been applied for thousands of years in China, though only used during the past three decades in the United States. ${ }^{11}$

Since TCM paradigm is unique from Western medicine due to its distinctive terminology and explanation, a brief description of TCM is provided for clarity and understanding of the
Correspondence: Joyce K. Anastasi, Director Division of Special Studies in Symptom Management, New York University, 380 Second Avenue, Suite 305, New York, NY 10010, USA.

Tel. +1.212.9927044 - Fax: +1.212.9953568.

E-mail: ja2188@nyu.edu

Key words: irritable bowel syndrome, IBS subtype constipation, traditional Chinese medicine, complementary and alternative medicine, acupuncture.

Contributions: JKA, Principal investigator of parent study, conceptualization of manuscript, interpretation, contributed to writing and editing of manuscript; BC, Clinical advisor, interpretation, contributed to writing and editing of manuscript; JQ, data management and analysis, contributed to writing and editing of manuscript; DM, analysis and interpretation, contributed to writing and editing of manuscript; CS, subject screening, study coordination, editing of manuscript.

Conflict of interests: the authors report no potential conflict of interests.

Funding: this work was supported by a grant from the NIH (R01NR010730).

Received for publication: 30 September 2011.

Revision received: 9 May 2012.

Accepted for publication: 14 May 2012.

This work is licensed under a Creative Commons Attribution NonCommercial 3.0 License (CC BYNC 3.0).

(c) Copyright J.K. Anastasi et al., 2012

Licensee PAGEPress, Italy

Gastroenterology Insights 2012; 4:e16

doi:10.4081/gi.2012.e16

framework. TCM is based on the concept that a balanced, unblocked flow of vital life energy in the body is required for health. This vital energy is called qi, which travels along 14 distinct pathways called channels or meridians. ${ }^{13} \mathrm{Qi}$ can be accessed through distinctive points along the channels allowing the vital energy to be restored, reduced, moved, and redirected to create a balanced flow of energy. There are a number of treatment modalities within TCM that aim to balance the flow of qi, including acupuncture, which involves the insertion of thin needles at specific points on the body, and moxibustion, the burning of moxa, or mugwort leaf, over specific acupuncture points. Acupuncture and moxibustion are often combined in the treatment of various symptoms.

In conducting our current randomized clinical trial focusing on IBS diarrhea subtype, our study recruitment team has received an overwhelming response from patients who fit the criteria for the constipation subtype. Due to this large response and patient interest, here we explore the characteristics of IBS-C 
patients from our current screening of adult patients who have presented with IBS according to ROME III criteria.

\section{Materials and Methods}

Subjects were recruited for our current study, Protocol vs. Patient-Oriented TCM Practices: A RCT for IBS Symptom Management (Diarrhea subtype). This is an ongoing 24week, double-blind, randomized, controlled, clinical trial investigating the efficacy of acupuncture and moxibustion in reducing the severity of abdominal pain and discomfort in patients with IBS-D (this study was approved by the Institutional Review Board at New York University School of Medicine). Recruitment materials, including newspaper and magazine advertisements and flyers, announcing our study for the treatment of IBS symptoms are distributed throughout the New York City metropolitan area. Individuals contacting our office regarding their interest in study participation and provider referrals are screened by our research staff. Responses to recruitment efforts from July 2009 to July 2011, which includes basic demographic characteristics, reported symptoms, triggers, medication use, current CAM use, and history of acupuncture use are summarized here.

\section{Results}

From July 2009 to July 2011, the screening of 1082 people revealed 380 (35\%) did not meet ROME III criteria for IBS and 702 (65\%) patients that reported a specific IBS subtype. Of these patients, there were 303 (43\%) with IBS-D and 272 (39\%) individuals with IBS-C. The IBS-C patients did not meet our study eligibility criteria due to the subtype (constipation) (Figure 1). Table 1 shows that the majority of the IBS-C patients were female (72\%), which approximates the proportion reported in epidemiological samples. ${ }^{4,8}$ To date, the mean age of patients reporting an IBS-C subtype is 45.3 years with a range of 18-80 years.

of the 272 patients identified with IBS-C, $38 \%$ of IBS-C patients were White, $31 \%$ were African American/Black, and nearly 15\% reported their race as 0ther. The most commonly reported IBS symptoms were bloating (52\%) and gas/flatulence (50\%). These patients reported stress and diet as the most common IBS triggers and acid reflux as the most common concurrent medical condition. The use of laxatives and stool softeners were reported by $15 \%$ and $13 \%$ of patients respectively. OTC drug use was reported in $25 \%$ of IBS-C patients, while prescription drug use

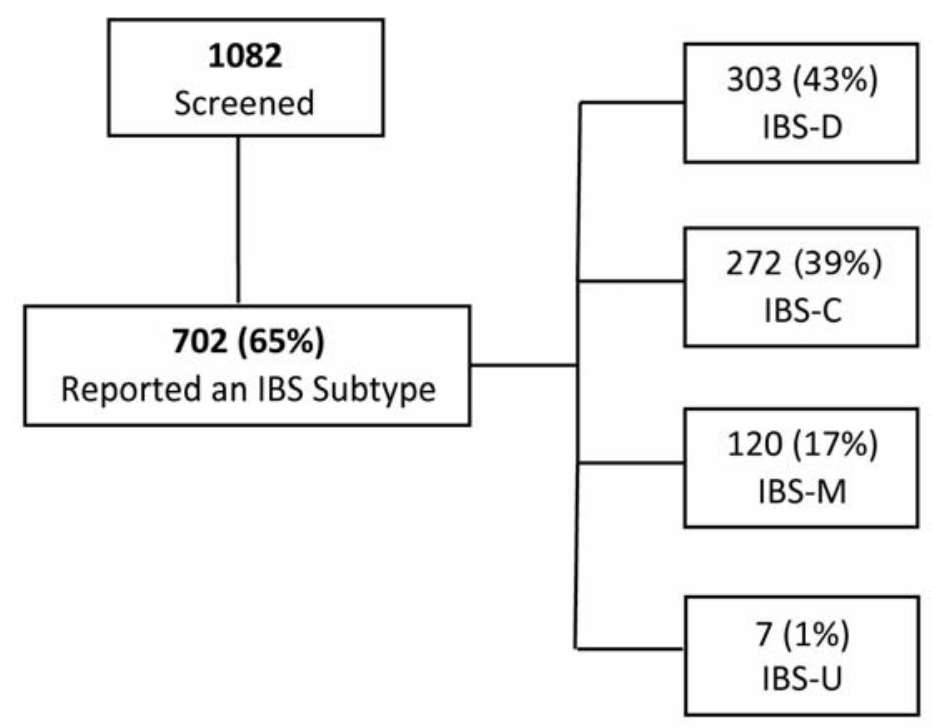

Figure 1. Patient screening flowchart (from July 2009 to July 2011).

Table 1. Irritable bowel syndrome constipation-subtype: patient characteristics $(\mathrm{N}=272)$.

\begin{tabular}{|c|c|c|}
\hline \multicolumn{3}{|l|}{ Age } \\
\hline Range & $18-80$ & \\
\hline Mean & 45.3 & \\
\hline Gender & $\mathbf{N}$ & $\%$ \\
\hline Male & 59 & 21.7 \\
\hline Female & 196 & 72.1 \\
\hline Not reported & 17 & 6.2 \\
\hline \multicolumn{3}{|l|}{ Ethnicity } \\
\hline Hispanic/latino & 40 & 14.7 \\
\hline Not hispanic/latino & 193 & 71.0 \\
\hline Not reported & 39 & 14.3 \\
\hline \multicolumn{3}{|l|}{ Race } \\
\hline African american/black & 83 & 30.5 \\
\hline White & 104 & 38.2 \\
\hline Asian & 7 & 2.6 \\
\hline American indian/Alaska native & 1 & 0.4 \\
\hline Other & 39 & 14.3 \\
\hline Not reported & 38 & 14.0 \\
\hline \multicolumn{3}{|l|}{ Medication (>3 days/week) } \\
\hline Any & 73 & 26.8 \\
\hline Laxatives & 42 & 15.4 \\
\hline Stool softeners & 35 & 12.9 \\
\hline Antidiarrheals & 2 & 0.7 \\
\hline Antispasmodics & 2 & 0.7 \\
\hline Probiotics & 9 & 3.3 \\
\hline Antidepressants & 4 & 1.5 \\
\hline Iron supplements & 3 & 1.1 \\
\hline Antibiotics & 1 & 0.4 \\
\hline Narcotics & 1 & 0.4 \\
\hline \multicolumn{3}{|l|}{ Symptoms } \\
\hline Gas/flatulence & 135 & 49.6 \\
\hline Bloating & 140 & 51.5 \\
\hline
\end{tabular}


and probiotic use were lower at $3 \%$ each. Patients most frequently reported that they were prescribed Amitiza, Bentyl, Nexium, and Wellbutrin. The majority of IBS-C patients (73\%) are not using medications to manage their symptoms. Few IBS-C patients reported current use of CAM and very few had ever received any type of acupuncture (Table 2).

\section{Discussion}

Irritable bowel syndrome, a chronic disorder, greatly impacts the lives of those who suffer from it and increases financial burden on individuals and the healthcare system. ${ }^{5}$ It is estimated that nearly a quarter of the population suffers from this debilitating condition involving abdominal pain, discomfort and interruptions in normal bowel function. ${ }^{1,4}$ The wide range of estimated IBS prevalence in North America (3\% to 20\%) is indicative of the varying case definitions and source populations studied. ${ }^{5}$ We use the ROME III criteria for IBS, as recommended to clinicians and researchers, to standardize the diagnosis of this functional bowel disease.

\section{Irritable bowel syndrome and quality of life}

The impact of IBS on quality of life (QOL) is considerable in terms of work absenteeism, social interactions and even simple everyday activities. ${ }^{5-7}$ Based on absences and impaired productivity, it is estimated that employees suffering from IBS lose approximately 14 hours of productivity during any given 40-hour workweek. ${ }^{5}$ In terms of QOL, studies have found that IBS patients have the same physical health-related quality of life (HRQOL) as patients with diabetes and an even lower physical HRQOL than patients who have depression or gastroesophageal reflux disease. ${ }^{5}$ Mental HRQOL is also affected by IBS. Symptoms of lowered mental HRQOL are associated with irregularities in sexual function, mood, and anxiety and are rooted in chronic stress and exhaustion, ${ }^{5}$ common complaints of those suffering from IBS. Patients report that these symptoms result in avoidance of socially vulnerable situations, like being away from restrooms, and activities such as eating out for dinner. ${ }^{5}$ In some cases, this reduction of HRQOL in IBS patients can be severe enough to increase the risk of suicidal behavior. ${ }^{5}$ Though these findings come from studies conducted in tertiary care referral populations, and therefore may not be applicable to community-based populations, IBS undeniably has a negative impact on HRQOL and this potential risk should not be overlooked. ${ }^{5}$ Comorbidities are common in patients who suffer from IBS.
Estimates of psychiatric comorbidities are as great as $90 \%$ of patients with IBS and chronic syndromes such as fibromyalgia, headaches, dyspepsia and chest pain are often reported by those suffering from IBS. ${ }^{9,12}$ The severity with which IBS affects QOL highlights the need to define and test treatments that are effective in improving this patient-oriented outcome. ${ }^{5,6,14}$

\section{Patient characteristics}

From July 2009 to July 2011 there were 1082 responses to recruitment efforts for our current study, of which $65 \%$ reported a specific IBS subtype. Of the individuals reporting a subtype, a substantial 39\% reported IBS-C. Though this number alone is meaningful, there are a number of studies that support our experience, reporting IBS-C in over $50 \%$ or even $75 \%$ of subjects. ${ }^{4}$ The majority of advertising and promotional materials distributed during subject recruitment and provider referrals throughout the course of our study are aimed at individuals with diarrhea-subtype IBS. However, so far, we screened nearly one half of patients reporting a subtype with IBS-C. It is likely that if advertising and promotional materials, as well as provider referrals, targeted individuals with constipation-subtype IBS, the number of IBS-C patients would be even higher and closer to the estimates seen in other studies. ${ }^{4}$

The majority of our screened IBS-C patients were female. Women are repeatedly found to have a higher prevalence of IBS than men in the literature. ${ }^{4,6,8}$ It has been shown that Caucasians appear to show a higher prevalence of IBS than African-Americans. ${ }^{15}$ Our findings are consistent with this observation, though we still saw a considerable number of AfricanAmerican patients as well as those reporting race as Other. A major benefit of being located in the New York City metropolitan area is the extremely diverse population from which subjects can be obtained, resulting in a study sample that is likely to better represent minority populations. Among the IBS-C patients we screened, in addition to abdominal pain, symptoms of bloating and gas/flatulence were commonly reported, as were stress and diet as the most common symptom triggers. These symptoms and triggers are often seen among IBS patients. ${ }^{6}$ Stress-induced exacerbations of symptoms tend to occur more frequently and severely in those who suffer from IBS. ${ }^{15}$ Furthermore, studies have found that individuals with IBS seem to have a lower threshold for coping with stressful situations and are more apt to react in ways which induce symptoms. ${ }^{15}$

\section{Drug and complementary and alternative medicine use}

Among the IBS-C patients we identified, one quarter reported OTC drug use, primarily laxa-

Table 2. Drug, complementary and alternative medicine, and acupuncture use.

\begin{tabular}{lcc} 
& IBS-C & All subtypes \\
$(\mathrm{N}=702)$ & $170(24 \%)$ \\
OTC drug use & $68(25 \%)$ & $7(4 \%)$ \\
Current CAM & $0(0 \%)$ & $34(20 \%)$ \\
\hline Full body acupuncture & $2(3 \%)$ & $5(3 \%)$ \\
\hline Auricular acupuncture & $1(1.5 \%)$ & $64(38 \%)$ \\
\hline None & $65(95.5 \%)$ & $93(13 \%)$ \\
\hline Prescription drug use & $8(3 \%)$ & $4(4 \%)$ \\
Current CAM & $0(0 \%)$ & $30(32 \%)$ \\
\hline Full body acupuncture & $1(12.5 \%)$ & $4(4 \%)$ \\
\hline Auricular acupuncture & $2(25 \%)$ & $45(48 \%)$ \\
\hline None & $5(62.5 \%)$ & $70(10 \%)$ \\
\hline Probiotic use & $8(3 \%)$ & $8(11 \%)$ \\
Current CAM & $0(0 \%)$ & $28(40 \%)$ \\
Full body acupuncture & $1(12.5 \%)$ & $3(4 \%)$ \\
\hline Auricular acupuncture & $0(0 \%)$ & $32(46 \%)$ \\
\hline None & $7(87.5 \%)$ & $235(33 \%)$ \\
\hline No drug use & $199(73 \%)$ & $2(0.9 \%)$ \\
Current CAM & $2(1 \%)$ & $58(25 \%)$ \\
\hline Full body acupuncture & $7(3.5 \%)$ & $21(9 \%)$ \\
\hline Auricular acupuncture & $0(0 \%)$ & $156(66 \%)$ \\
\hline None & $191(96 \%)$ & \\
\hline
\end{tabular}

CAM, complementary and alternative medicine; IBS-C, irritable bowel syndrome constipation-subtype; OTC, over-the-counter. Percentages may not add up to $100 \%$ due to the fact that a single study subject could potentially be included in multiple categories. 
tives and stool softeners, while prescription drug use and probiotic use were seldom reported.OTC drug use patterns of IBS-C patients were similar to those of all patients combined; however prescription drug use and probiotic use were higher in all of the other IBS-subtype patients combined than in IBS-C patients alone. Compared to non-IBS-C patients, those with IBS-C were much less likely to report use of medications to manage symptoms. Current reported use of CAM was lower across all subtypes than the reported use of CAM in 35\% to 50\% of patients suffering from functional bowel diseases, including IBS, that has been found in the US. ${ }^{6,11}$ The proportion of CAM use among the non-IBS-C subtypes was higher overall and highest in patients using probiotics. Among IBS-C patients, previous full body acupuncture experience was significantly lower than the other IBS subtypes. Previous auricular acupuncture experience was substantially lower than full body acupuncture across all subtypes, but again, much lower among IBS-C patients. The proportion of those with previous full body acupuncture experience was highest among patients using probiotics for both IBS-C patients alone and all of the other IBS-subtype patients combined. Previous auricular acupuncture was highest among IBS-C patients using prescription drugs and among all patients combined reporting no medication use. These findings suggest that few IBS-C patients seek professional attention for their symptoms and very few regularly use any form of treatment for symptom relief. The majority of those patients who selftreat with OTC drugs find they lead to unsatisfactory results. This is especially true of IBS-C patients. However, the number of inquiries we received and subsequent screenings we performed highlight the level of interest in acupuncture as a treatment option for individuals suffering from this IBS constipation subtype.

\section{Implications and recommendations}

Since a large portion of the population lives with IBS-C, effective treatment options are needed. Information specific to IBS subtype and the recruitment of these subjects, as explored in this paper, is important to the advancement of the field. The considerable number of IBS-C patients identified, which is likely conservative, the limited conventional treatment options and the possible benefits of CAM approaches to manage IBS symptoms, requires further research. ${ }^{9,10,16,17}$

\section{References}

1. Drossman DA. Rome III: the functional gastrointestinal disorders. $3^{\text {rd }}$ ed. McLean, VA: Degnon Associates Inc.; 2006.

2. Spiegel BMR, Bolus R, Harris LA, et al. Characterizing abdominal pain in IBS: guidance for study inclusion criteria, outcome measurement and clinical practice. Aliment Pharmacol Ther 2010;32:1192202.

3. Furman DL, Cash BD. The role of diagnostic testing in irritable bowel syndrome. Gastroenterol Clin N Am 2011;40:105-19.

4. Grundmann 0, Yoon SL. Irritable bowel syndrome: epidemiology, diagnosis and treatment: an update for health-care practitioners. J Gastroenterol Hepatol 2010;25: 91-9.

5. Agarwal N, Spiegel BMR. The effect of irritable bowel syndrome on health-related quality of life and health care expenditures. Gastroenterol Clin N Am 2011;40:119.

6. Yoon SL, Grundmann 0, Koepp L, Farrell L. Management of irritable bowel syndrome (IBS) in adults: conventional and complementary/alternative approaches. Altern Med Rev 2011;16:134-51.

7. Heitkemper M, Olden K, Gordon S, et al. Irritable bowel syndrome: a survey of nurses' knowledge. Gastroenterol Nurs 2001; 24:281-7.

8. Talley NJ, Zinmeister AR, Melton LJ $3^{\text {rd }}$. Irritable bowel syndrome in a community: symptom subgroups, risk factors, and health care utilization. Am J Epidemiol 1995;142:76-83.

9. Friedrich M, Grady SE, Wall GC. Effects of antidepressants in patients with irritable bowel syndrome and comorbid depression. Clin Ther 2010;32:1221-33.

10. National Center for Complementary and Alternative Medicine, National Institutes of Health, US Department of Health and Human Services. Irritable Bowel Syndrome and CAM: at a glance. July 2010. Updated October 2010. 1-6. Available from: http:// nccam.nih.gov/sites/nccam.nih.gov/files/lB S_D452.pdf

11. Magge S, Lembo A. Complementary and alternative medicine for the irritable bowel syndrome. Gastroenterol Clin N Am 2011;40:245-53.

12. van Tilburg MA, Palsson OS, Levy RL, et al. Complementary and alternative medicine use and cost in functional bowel disorders: a six month prospective study in a large HMO. BMC Complement Altern Med 2008; 8:46.

13. Riddle J. Report of the New York State Commission on acupuncture. Am J Chin Med 1974;2:289-318.

14. Voci SC, Cramer KM. Gender-related traits, quality of life, and psychological adjustment among women with irritable bowel syndrome. Qual Life Res 2009;18:1169-76.

15. Pae CU, Masand PS, Ajwani N, et al. Irritable bowel syndrome in psychiatric perspectives: a comprehensive review. Int J ClinPract 2007;61:1708-18.

16. Reynolds JA, Bland JM, MacPherson H. Acupuncture for irritable bowel syndrome an exploratory randomised controlled trial. Acupunct Med 2008;26:8-16.

17. Anastasi JK, McMahon DJ, Kim, GH. Symptom management for irritable bowel syndrome: a pilot randomized controlled trial of acupuncture/moxibustion. Gastroenterol Nurs 2009;32: 243-55. 